Research Paper

\title{
Temozolomide and Bevacizumab Induction before Chemoradiotherapy in Patients with Bulky Glioblastoma and/or with Severe Neurological Impairment
}

\author{
Ilan Darmon'1, Mony Chenda Morisse², Alexandre Coutte ${ }^{1}$, Marie Blonski³, Emilie Le Rhun', Luc \\ Taillandier 3 , Diana Bello Roufai ${ }^{2}$, Christine Desenclos 5 , Stéphanie Trudel 6 , Jean-Christophe Faivre ${ }^{7,8}$, \\ Nicolas Blanchard ${ }^{9}$, Bruno Chauffert ${ }^{2}$, Mathieu Boone ${ }^{2 \bowtie}$ \\ 1. Department of Radiotherapy, University Hospital, Amiens, France; \\ 2. Department of Medical Oncology , University Hospital, Amiens, France; \\ 3. Department of Neuro-Oncology, University Hospital, Nancy, France; \\ 4. Department of Neuro-Oncology, University Hospital, Lille, France; Department of Neurology, General Hospital, Valenciennes, France; Neurology, Breast \\ Unit, Oscar Lambret, Lille, France; INSERM UMR 1192, Laboratoire de Protéomique, Réponse Inflammatoire, Spectrométrie de Masse (PRISM), Lille \\ University, Villeneuve d'Ascq, France; \\ 5. Department of Neurosurgery, University Hospital, Amiens, France; \\ 6. Laboratory of Molecular Oncology, University Hospital, Amiens, France; \\ 7. Department of Radiotherapy, Centre Alexis Vautrin, Nancy, France; \\ 8. Department of Radiotherapy, Hospital of Metz-Thionville, France; \\ 9. Department of Radiotherapy, Clinique des Dentellières, Valenciennes, France.
}

$\square$ Corresponding author: Mathieu Boone, MD. Department of Medical Oncology, University Hospital, Amiens, France 80054 Amiens Cedex 1 Phone: +33 322 455499 Fax: +33 322456091 Email: boone.mathieu@chu-amiens.fr

(c) Ivyspring International Publisher. This is an open access article distributed under the terms of the Creative Commons Attribution (CC BY-NC) license (https:// creativecommons.org/licenses/by-nc/4.0/). See http://ivyspring.com/terms for full terms and conditions.

Received: 2016.11.14; Accepted: 2017.02.08; Published: 2017.05.12

\begin{abstract}
Background. New approaches are needed for patients newly diagnosed with bulky glioblastoma (GB) and/or with severe neurological impairment that cannot benefit from first line temozolomide (TMZ)-based chemoradiotherapy. Bevacizumab (BEV), an antiangiogenic anti-VEGF-R monoclonal antibody, has a rapid impact on tumor-related brain edema in recurrent GB. The present study reports the feasibility and efficacy of an induction treatment with TMZ and BEV to alleviate the initial neurological impairment and/or to reduce the tumor volume before a delayed chemoradiotherapy.

Methods. We retrospectively analyzed tumor and target volumes and clinical neurological status in 39 patients with bulky GB and/or with severe neurological impairment after an induction treatment combining TMZ and BEV. Neurological and radiological responses were assessed according to RANO criteria. Calculating gross tumor and clinical target volumes (GTV and CTV) was done at diagnosis and before radiotherapy. Progression-free survival (PFS) and overall survival (OS) were determined by Kaplan Meier methods. Safety was reported according to NCTCAE.

Results. A cohort of 39 patients was analyzed between December 2010 and April 2014. Upfront standard TMZ-based chemoradiotherapy was recused due either to tumor volume or impairment of neurological status and/or performance status. After TMZ/BEV induction (median time of 3 months), 6 (15\%) patients achieved a partial response (PR), and 17 (44\%) had a stable disease. 24 patients (62\%) received a radical-intent chemoradiotherapy. TMZ-BEV induced median reduction of the clinical target volume (CTV) was $25.9 \%$ [-84.4\%; - 4.8\%]. The median PFS and OS were 8.4 months [95\% Cl: $(6.6-9.9)$ ] and 11.0 months [95\% Cl: $(9.3$ - 13.7)], respectively in the whole cohort and 10.8 [95\% Cl: $(9.3-12.9)$ ] and 15.0 [95\% Cl: (I3.2 - I7.8)] for irradiated patients. Induction treatment led to corticosteroid dose reduction or cessation in 21 patients (54\%). KPS improvement was observed in $38 \%$ of patients. Toxicity was mild with only $7 / 39$ (18\%) grade III-IV toxicity, including 1 digestive bleeding and 1 epistaxis.

Conclusion. TMZ-BEV induction led to CTV reduction allowing for optimal chemoradiotherapy in a majority (62\%) of patients for which radiotherapy was initially recused. A clinical benefit was obtained with improved KPS and a decrease in steroid dose.
\end{abstract}

Key words: bulky glioblastoma, multifocal glioblastoma, bevacizumab, temozolomide, radiotherapy. 


\section{Introduction}

Glioblastoma (GB; WHO grade IV gliomas) is the most common malignant primitive brain tumor. Patients are classified for prognosis using a simplification of the original recursive partitioning analysis (RPA) classification from the RTOG [1]. Three distinct prognostic classes (RPA III, IV, and V) are defined by age, performance status, extent of resection and neurological status. The standard treatment for RPA III and IV patients is surgical resection when possible, followed by radiotherapy and concomitant, then adjuvant temozolomide (TMZ) [2]. This regimen prolongs overall survival in comparison with radiotherapy alone. However, a large proportion of patients (222/484, i.e. $46 \%$ in the Li study) with newly diagnosed GB are ranked in the RPA class V due to neurological or cognitive impairment, and/or biopsy alone and/or a Karnofsky score $<70$ (RPA V). Prognosis is poor for RPA class $\mathrm{V}$ patients with a median survival time of 6.7 months, compared to 17.1, and 11.2 months, for RPA classes III and IV, respectively. Moreover, patients with bulky and/or multifocal disease and/or in poor general condition may not even receive radiotherapy and are generally not included in clinical trials. Recent guidelines recommend $\mathrm{TMZ}$ as monotherapy or best supportive care for these patients, especially with a Karnofsky score under 50 [3]. GB is a vascularized tumor, overexpressing Vascular Endothelial Growth Factor A (VEGF-A) [4]. Bevacizumab (BEV) is a monoclonal antibody that traps VEGF and has an antiangiogenic activity. BEV has been introduced for recurrent GB after the failure of standard TMZ-based chemoradiotherapy [5]. Impressive and fast responses are often observed, leading to the accelerated registration of BEV by the FDA (Food and Drug Administration) in the USA but not by EMA (European Medicines Agency) in Europe. The AVAglio (Avastin in Glioblastoma) and RTOG-0825 randomized, placebo-controlled phase III trials in newly diagnosed RPA class III and IV GB reported prolonged progression-free survival (PFS), but not longer overall survival (OS), with the addition of BEV to radiotherapy and TMZ [6, 7]. An increased PFS (7.1 months versus 5.2 months), but not OS (11.3 months) was also reported after neo-adjuvant and adjuvant $\mathrm{BEV} /$ irinotecan combined with TMZ-radiation in the first-line treatment of unresectable GB [8]. However, none of these trials included patients with bulky GB, and/or with severe neurological impairment at diagnosis despite the hypothesis that BEV could be especially efficient in this situation. We reported here a series of patients in whom radiotherapy was considered as not feasible in first intention but most of which could be treated by optimal chemoradiotherapy after upfront TMZ and BEV treatment. We analyzed the impact of this induction strategy on tumor and target volumes, neurological status and survival.

\section{Patients and Methods}

\section{Patients}

A common therapeutic strategy was used between December 2010 and April 2014 by three French neuro-oncology teams (Amiens, Nancy, and Valenciennes) for newly diagnosed GB patients with bulky and/or multifocal disease, and/or with severe neurological impairment that could not be treated upfront with a standard TMZ-based chemoradiotherapy. We retrospectively collected clinical and radiological data from medical files. Patients were over 18 years old. Most had histologically proven unresectable primary GB. In some cases, GB diagnosis was only done by multimodal MRI with confirmation by a collegial agreement including an expert in neuroradiology. Radiotherapy in first intention was ruled out either due to tumor volume, and/or due to severe neurological impairment (MRC neurological status $\geq$ 3) [9], and/or poor performance status (KPS $<70 \%$ ), despite the use of corticosteroids at $\geq 0.5 \mathrm{mg} / \mathrm{kg}$ prednisone equivalent-dose, according to a multidisciplinary team decision. Some patients with early and rapid progression after a surgical resection ( $<28$ days) were also included. Patients with separate multifocal GB (hemispheric bilateral disease) were not included. The biological conditions had to be sufficient to allow the use of TMZ and BEV. Patients received information about the off-label use of BEV in Europe, potential BEV and TMZ adverse events, and gave their informed consent.

\section{Treatments}

For the induction phase, patients received TMZ per os, $150 \mathrm{mg} / \mathrm{m} 2 /$ day X 5 days for the first cycle, then $200 \mathrm{mg} / \mathrm{m} 2 /$ day X 5 days for the other monthly cycles if well tolerated. Intravenous BEV, 10 $\mathrm{mg} / \mathrm{kg} /$ day, was IV given every 2 weeks. Patients were restaged every 6 to 8 weeks by the multidisciplinary team. Radiotherapy was started when a maximal or at least stable response was obtained considering MRI (Magnetic Resonance Imaging) results and the clinical status. Radiotherapy was carried out by an intensity-modulated radiotherapy (IMRT) technique or a 3D conformal radiotherapy (3D-CRT) according to the hospital facilities. IMRT was performed either with a static method (step and shoot) or a rotational one (volumetric modulated arc therapy; VMAT). Target delineation was performed according to EORTC 
(European Organization for Research and Treatment of Cancer) guidelines [10]. CT (computed tomography) scan scheduling was fused with MRI assessment that was performed after the induction phase. Prescribed radiation dose ranged from 54 to 60 Gy in 1.8 to 2 Gy fraction, taking into account organs at risk (ORs) proximity and dosimetric constraints in the radiotherapy planning. Clinical target volume (CTV) was typically the gross tumor volume (GTV) of enhancing regions on T1-sequences, with the addition of a $20 \mathrm{~mm}$ margin. Planning target volume (PTV) margin around CTV varied from 3 to $5 \mathrm{~mm}$ depending on centers. Radiotherapy planning took into account dose constraints to ORs according to guidelines: $1 / 3$ of brain should not receive $>60 \mathrm{~Gy}$, $2 / 3$ of brain should not receive $>50 \mathrm{~Gy}$, the whole brain should not receive $>45 \mathrm{~Gy}$, and brain stem, optic nerves and optic chiasm should not receive $>54 \mathrm{~Gy}$ $[10,11]$. In order to allow for comparisons, CT scans were also fused with MRI performed before induction phase (T1 with gadolinium+ T2 FLAIR) to determine tumor and target volumes (GTV, CTV and PTV), as they should have been defined in absence of the induction phase. Patients received TMZ per os, 75 $\mathrm{mg} / \mathrm{m}^{2}$ /day from the first to the last day of radiotherapy. Most patients continued to receive $\mathrm{BEV}$, $10 \mathrm{mg} / \mathrm{kg} /$ day every 2 weeks, during radiotherapy. TMZ dose was 150 à $200 \mathrm{mg} / \mathrm{m} 2 /$ day for 5 days for every 28-day cycle for the adjuvant phase, and BEV was continued if tolerated, either at $10 \mathrm{mg} / \mathrm{kg} /$ day every 2 weeks or at $15 \mathrm{mg} / \mathrm{kg} /$ day every 3 weeks. Treatment was continued until progression or toxicity.

\section{Assessment of clinical and radiological response}

OS and PFS were measured from the date of initial diagnosis (date of biopsy or date of the first MRI if biopsy was not done), and were determined using the Kaplan-Meier method.

Initial MRI included T1 weighted sequences, with and without gadolinium injection, T2, T2 FLAIR and when possible, diffusion and perfusion weighted sequences and spectroscopy. MRI was repeated every 6-8 weeks during the induction phase. Response was determined according to RANO criteria [12]. Toxicity was assessed using the CTCAE v 4.03. Corticosteroid dose, KPS (Karnofsky Performance Status) and MRC (Medical Research Council) neurological status were obtained from the medical records.

\section{Statistical analysis}

Analyses were carried out using the R software version 3.0.1. OS and PFS were determined using the Kaplan-Meier method.

\section{Results}

\section{Patient characteristics}

The study involved 39 patients that received upfront TMZ and BEV for GB from December 2010 to April 2014 (Figure 1, Table 1). The median age was 60 and the sex ratio was 1.3 . Most patients $(74 \%)$ were classed in RPA class V. Some patients were first in RPA class III and IV after surgical resection (23\%), but disease rapidly recurred before radiotherapy. Most patients (74\%) had undergone only biopsy. GB diagnosis was done only by imaging in three patients due to intracranial hypertension. Tumor was bifocal at presentation in 15 patients $(38 \%)$, but could be finally included in a unique radiation field. In the rest of the patients $(59 \%)$, the tumor was unifocal, but its volume was considered as being too large for an upfront irradiation and/or there was a neurological impairment and/or intracranial hypertension. Most patients $(87 \%)$ received an initial steroid dose $>0.5$ $\mathrm{mg} / \mathrm{kg}$ of prednisone-equivalent.

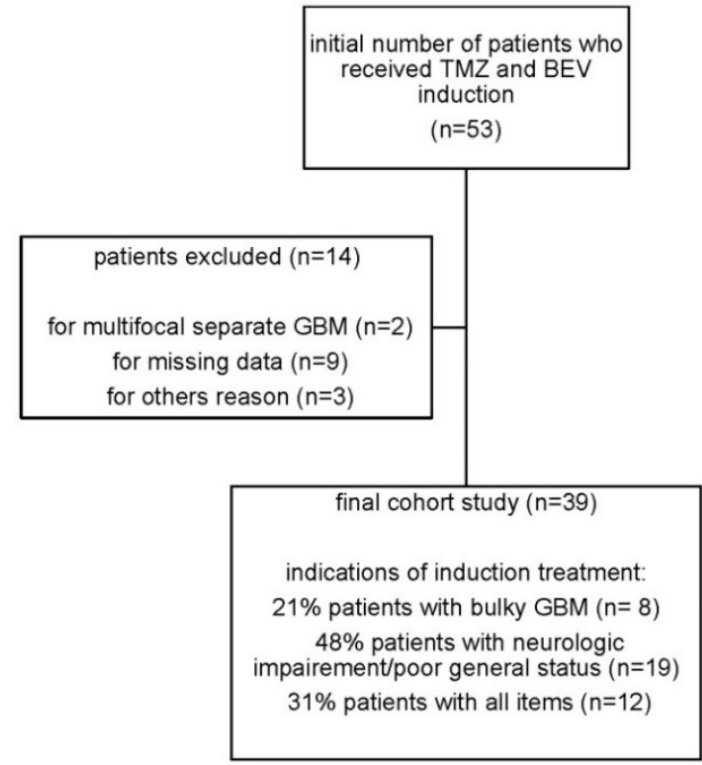

Figure 1. Flowchart of the study

\section{Treatment}

All patients who received at least one cycle of TMZ and BEV as induction treatment are shown in Table 2. Median number of monthly TMZ cycles was 3 (range 1 to 12) and median number of BEV injections was 6 (range 1 to 20). Some patients received $\mathrm{TMZ} / \mathrm{BEV}$ for a longer period of time before the decision to proceed to radiotherapy. Median time from $\mathrm{TMZ} / \mathrm{BEV}$ induction treatment to radiotherapy was 3,2 months (range from 1,8 months to 12,9 months). Overall 24 patients (62\%) with partial response or stable disease received a delayed complete (60 Gy) radiotherapy (Table 2). No radiation 
therapy had to be stopped due to disease progression. The other 15 patients (38\%) did not receive chemoradiotherapy due to neurological deterioration or radiological progression during the induction time. After the induction phase, they were treated by chemotherapy alone or with supportive care. Concomitant drugs during radiotherapy were given depending on the clinician's choice. Most patients received daily TMZ, $75 \mathrm{mg} / \mathrm{m} 2 / \mathrm{d}$ and BEV every 2 weeks (16/24). TMZ alone was given in 7 patients and $\mathrm{BEV}$ alone in 1 patient, due to a grade IV TMZ-induced neutropenia. Most patients continued to receive monthly $\mathrm{TMZ}$ and $\mathrm{BEV}$ in the post-radiotherapy period until disease progression and/or toxicity.

Table 1. Characteristics of the study population $\mathrm{N}=39$

\begin{tabular}{|c|c|}
\hline Age, years, median (range) & $60(22-77)$ \\
\hline \multicolumn{2}{|l|}{ Age, $\mathrm{n}(\%)$} \\
\hline$<50$ year-old & $8(21 \%)$ \\
\hline$>$ 50year-old & $31(79 \%)$ \\
\hline Sex, M/F, n (\%) & $22(56 \%) / 17(44 \%)$ \\
\hline \multicolumn{2}{|l|}{ WHO performance status (PS), n (\%) } \\
\hline 0 & $7(18 \%)$ \\
\hline 1 & $13(33 \%)$ \\
\hline 2 & $9(23 \%)$ \\
\hline 3 & $5(13 \%)$ \\
\hline 4 & $1(3 \%)$ \\
\hline UNK & $4(10 \%)$ \\
\hline \multicolumn{2}{|l|}{ KPS at initial diagnosis, $\mathbf{n}(\%)$} \\
\hline$\geq 70$ & $20(51 \%)$ \\
\hline$<70$ & $15(38 \%)$ \\
\hline UNK & $4(10 \%)$ \\
\hline \multicolumn{2}{|l|}{ RPA class, n (\%) } \\
\hline III & $2(5 \%)$ \\
\hline IV & $7(18 \%)$ \\
\hline $\mathrm{V}$ & $29(74 \%)$ \\
\hline UNK & $1(3 \%)$ \\
\hline \multicolumn{2}{|l|}{ Tumor presentation, $\mathbf{n}(\%)$} \\
\hline Unifocal & $23(59 \%)$ \\
\hline Multifocal & $15(38 \%)$ \\
\hline UNK & $1(3 \%)$ \\
\hline \multicolumn{2}{|l|}{ Diagnosis modalities, $\mathbf{n}(\%)$} \\
\hline Biopsy & $29(74 \%)$ \\
\hline Surgery & $7(18 \%)$ \\
\hline Partial resection & $6(15 \%)$ \\
\hline Complete resection & $1(3 \%)$ \\
\hline Imaging & $3(8 \%)$ \\
\hline $\begin{array}{l}\text { Bevacizumab treatment, number of cycles, median } \\
\text { (range) }\end{array}$ & $7(1-20)$ \\
\hline $\begin{array}{l}\text { Upfront Temozolomide treatment, number of cycles, } \\
\text { median (range) }\end{array}$ & $3(1-12)$ \\
\hline $\begin{array}{l}\text { Upfront Bevacizumab treatment, number of cycles, } \\
\text { median (range) }\end{array}$ & $6(1-20)$ \\
\hline \multicolumn{2}{|l|}{ Initial corticosteroid, $\mathbf{n}(\%)$} \\
\hline Yes & $34(87 \%)$ \\
\hline No & $3(8 \%)$ \\
\hline UNK & $2(5 \%)$ \\
\hline $\begin{array}{l}\text { Initial corticosteroid dose (prednisone equivalent), mg, } \\
\text { median (range) }\end{array}$ & $60(0-150)$ \\
\hline \multicolumn{2}{|l|}{ Indication of induction treatment } \\
\hline bulky GBM & $8(21 \%)$ \\
\hline neurologic impairement/poor general status & $19(48 \%)$ \\
\hline all items & $12(31 \%)$ \\
\hline
\end{tabular}

UNK: unknown
Table 2. Treatment Modalities

\begin{tabular}{ll}
\hline & Median (range) \\
\hline Induction & $3(1-12)$ \\
Number of monthly TMZ cycles & $6(1-20)$ \\
Full radiotherapy (60 Gy) & $\mathrm{n}(\%)$ \\
Yes & $24(62 \%)$ \\
No (no radiotherapy) & $15(38 \%)$ \\
Technique & \\
3D-CRT & $11(46 \%)$ \\
IMRT & $12(50 \%)$ \\
UNK & $1(4 \%)$ \\
Concomitant radiochemotherapy regimen & $\mathrm{n}(\%$ in 24 patients) \\
TMZ+BEV & $16(67 \%)$ \\
TMZ alone & $7(29 \%)$ \\
BEV alone & $1(4 \%)$ \\
\hline
\end{tabular}

\section{Response to induction treatment}

According to the clinicians' report, neurological and performance status improved in 15 patients $(38 \%)$, was stable in $16(41 \%)$, or deteriorated in 8 $(21 \%)$ in the whole cohort of 39 patients (Table 4). Clinical and radiological response was retrospectively assessed by RANO criteria. No complete response was reported. Partial response was observed in 6 patients $(15 \%)$, stable response in $17(44 \%)$, and progression in 15 (38\%) (Table 4). Data were missing for one patient. Corticosteroids were stopped in 7 patients $(18 \%)$, or reduced in 14 patients $(36 \%)$, with a median reduction of $20 \mathrm{mg}$ in prednisone-equivalent dose (data not shown). Daily dose of corticosteroids was stable in 8 patients $(21 \%)$ or increased in 4 patients (10\%). Median corticosteroid dose was $60 \mathrm{mg}$ at initial diagnosis versus $20 \mathrm{mg}$ at time of induction assessment. Data were missing for 6 patients. Special attention was paid to the evolution of the tumor volume to be irradiated after induction (Table 4). This analysis was done in the 24 patients with stable or partial responses that received delayed radiotherapy. Induction treatment induced a global decrease of GTV, CTV and PTV. Decrease was of course more dramatic in the 6 patients that were in strict partial response according to RANO criteria. Irradiated normal brain volume represented by a PTV/whole brain ratio decreased from 0.33 to 0.25 in the global cohort of irradiated patients.

There was no difference in the initial characteristics between patients who have received radiotherapy or not, except for the corticosteroid dose (Table 3).

\section{Toxicity}

Toxicity was mild both during the TMZ/BEV induction and the chemoradiotherapy phases (Table 5). There were no treatment related-deaths. Grade III-IV toxicities were reported in 7 patients $(18 \%)$ during the induction phase. Most were hematological 
(3 thrombocytopenia, 1 neutropenia). Grade III-IV bleeding occurred in 2 patients $(1$ digestive hemorrhage related to a colon diverticulitis and 1 abundant epistaxis) leading to discontinuation of BEV. Hypertension was controlled by an appropriate treatment and BEV was continued in 1 patient. Only 1 grade III-IV hematological toxicity (thrombocytopenia with neutropenia in the same patient) was reported during the chemoradiotherapy phase leading to a definitive cessation of TMZ treatment for this patient.

\section{Survival}

Median PFS and OS in patients treated by upfront TMZ-BEV were respectively 8.4 months (95\% CI: [6.6 - 9.9]) and 11.0 months (95\% CI: [9.3 - 13.7]) in the whole cohort of 39 patients (Figure 2) and 10.8 (95\% CI: [9.3 - 12.9]) and 15.0 (95\% CI: [13.2 - 17.8]) in the 24 irradiated patients (Figure 3).

Table 3. Characteristics of patients who received or did not receive irradiation

\begin{tabular}{|c|c|c|c|}
\hline & & $\begin{array}{l}\text { Radiotherapy } \\
\mathrm{N}=24\end{array}$ & $\begin{array}{l}\text { No radiotherapy } \\
N=15\end{array}$ \\
\hline Age, years, median (range) & & $60(20-69)$ & $61(46-77)$ \\
\hline \multicolumn{4}{|l|}{ Age, n (\%) } \\
\hline$<50$ year-old & & $6(25 \%)$ & $2(13 \%)$ \\
\hline$>$ 50year-old & & $18(75 \%)$ & $13(87 \%)$ \\
\hline Sex, M/F, n (\%) & & $15(63 \%) / 9(37 \%)$ & $7(47 \%) / 8(53 \%)$ \\
\hline \multicolumn{4}{|l|}{$\begin{array}{l}\text { KPS at initial diagnosis, } \mathbf{n} \\
(\%)\end{array}$} \\
\hline$\geq 70$ & & $16(67 \%)$ & $4(27 \%)$ \\
\hline$<70$ & & $8(33 \%)$ & $7(46 \%)$ \\
\hline UNK & & 0 & $4(27 \%)$ \\
\hline \multicolumn{4}{|l|}{ RPA class, $n(\%)$} \\
\hline III & & $2(8 \%)$ & 0 \\
\hline IV & & $5(21 \%)$ & $2(13 \%)$ \\
\hline V & & $17(71 \%)$ & $12(80 \%)$ \\
\hline UNK & & 0 & $1(7 \%)$ \\
\hline \multicolumn{4}{|l|}{ Tumor presentation, $\mathrm{n}(\%)$} \\
\hline Unifocal & & $17(71 \%)$ & $6(40 \%)$ \\
\hline Multifocal & & $7(29 \%)$ & $8(53 \%)$ \\
\hline UNK & & 0 & $1(7 \%)$ \\
\hline \multicolumn{4}{|l|}{ Diagnosis modalities, $\mathrm{n}(\%)$} \\
\hline Biopsy & & $17(71 \%)$ & $12(80 \%)$ \\
\hline Surgery & & $6(25 \%)$ & $1(7 \%)$ \\
\hline Partial resection & & $5(21 \%)$ & $1(7 \%)$ \\
\hline Complete resection & & $1(4 \%)$ & 0 \\
\hline Imaging & & $1(4 \%)$ & $2(13 \%)$ \\
\hline $\begin{array}{l}\text { Upfront Temozolomide } \\
\text { treatment, number of cycles, } \\
\text { median (range) }\end{array}$ & $3(1-12)$ & $3(2-12)$ & $3(1-9)$ \\
\hline $\begin{array}{l}\text { Upfront Bevacizumab } \\
\text { treatment, number of cycles, } \\
\text { median (range) }\end{array}$ & $6(1-20)$ & $7(4-20)$ & $5(1-18)$ \\
\hline \multicolumn{4}{|l|}{ Initial corticosteroids, $\mathbf{n}(\%)$} \\
\hline Yes & & $21(87.5 \%)$ & $13(87 \%)$ \\
\hline No & & $3(12.5 \%)$ & 0 \\
\hline UNK & & 0 & $2(13 \%)$ \\
\hline $\begin{array}{l}\text { Initial corticosteroid dose } \\
\text { (mg prednisone equivalent) } \\
\text { median (range) }\end{array}$ & & $40(0-150)$ & $125(20-150)$ \\
\hline
\end{tabular}

In patients in RPA III/IV and RPA V classes, the median OS was 14.4 months (95\%CI: [11.2 -21.1]) and 8.4 months (95\%CI: [8.1 -12.7$]) ; p=0.217$ respectively and the median PFS was 12.1 months $(95 \% \mathrm{CI}$ : [9.4 -16.7]) and 7.1 months (95\%CI: [5.4 -8.5]); $p=0.01$ respectively.

Table 4. Evolution of radiotherapy parameters after TMZ-BEV induction

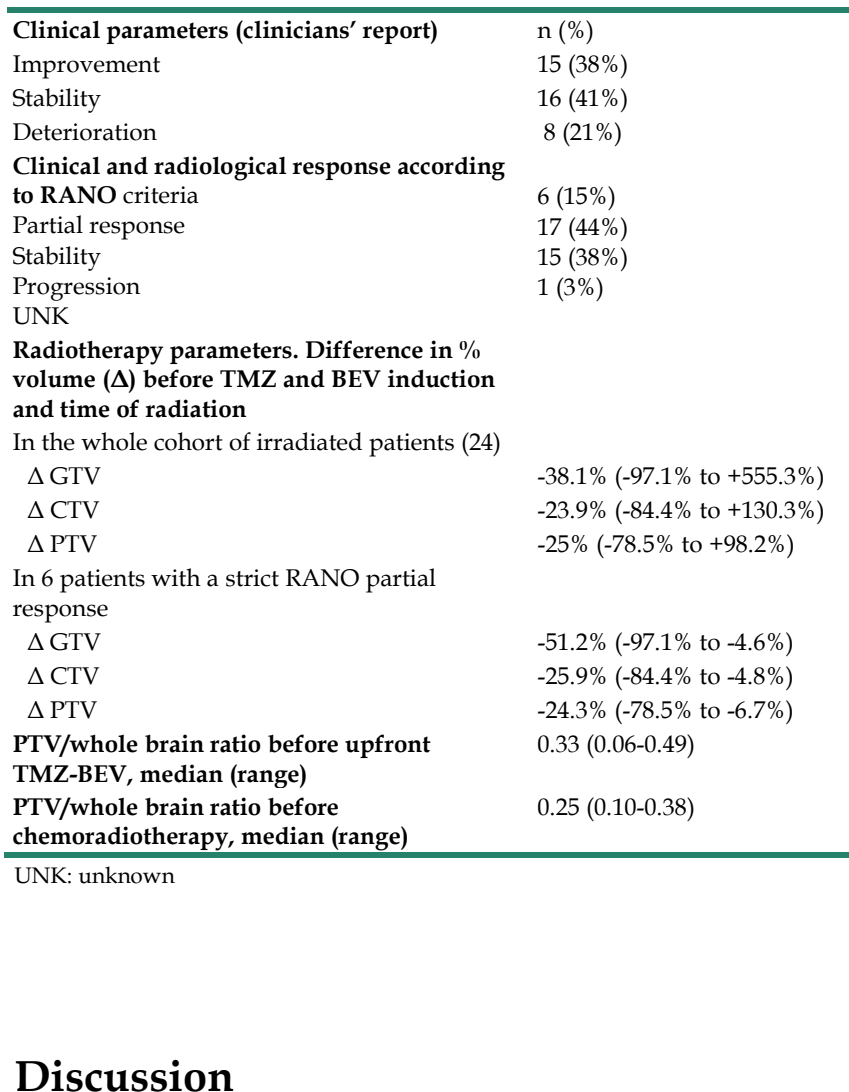

This study reviewed the results from three experienced centers that have used a similar strategy using TMZ-BEV induction in GB patients that could not be firstly treated by the classical TMZ-radiotherapy regimen, either due to excessive GB tumor volume and/or neurological impairments. TMZ/BEV induction was followed by a radical-intent chemoradiotherapy in responders and in patients with better performance status and stable disease on MRI. Remarkably, $62 \%$ of patients were able to complete a delayed standard radiotherapy (60 Gy) with concomitant TMZ, and in most cases with BEV continuation. 
Table 5. Adverse effects during upfront TMZ-BEV and during radiochemotherapy, $\mathrm{n}(\%)$

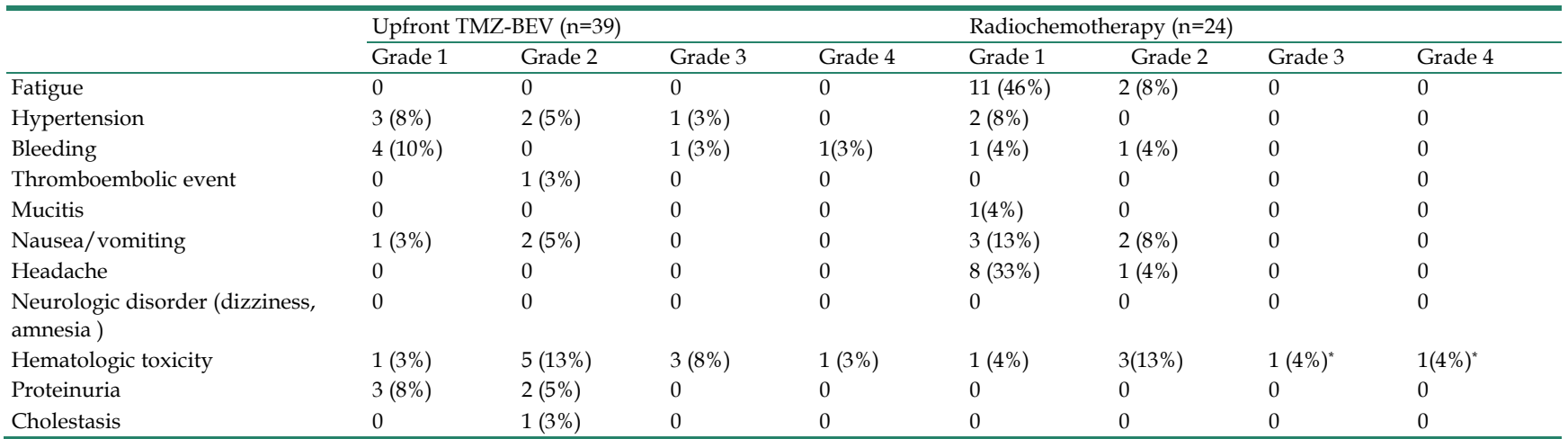

Missing data were considered as absence of adverse events. *: same patient with neutropenia grade 4 and thrombopenia grade 3

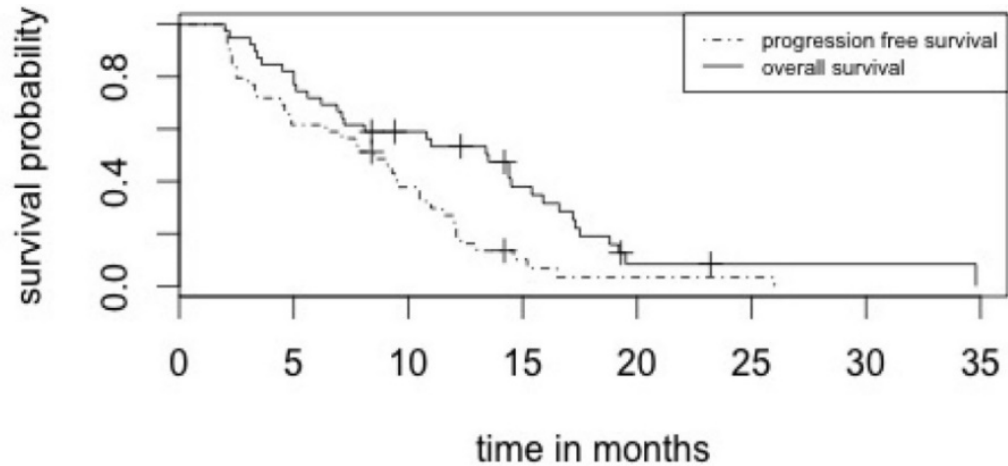

\begin{tabular}{|c|c|c|c|c|c|c|c|}
\hline $\begin{array}{c}\text { Progression-free } \\
\text { survival }\end{array}$ & 39 & 24 & 14 & 3 & 1 & 1 & 0 \\
\hline Overall survival & 39 & 30 & 21 & 12 & 2 & 1 & 1 \\
\hline
\end{tabular}

Figure 2. Progression free survival and overall survival for the whole cohort of 39 patients who received induction treatment by TMZ/BEV

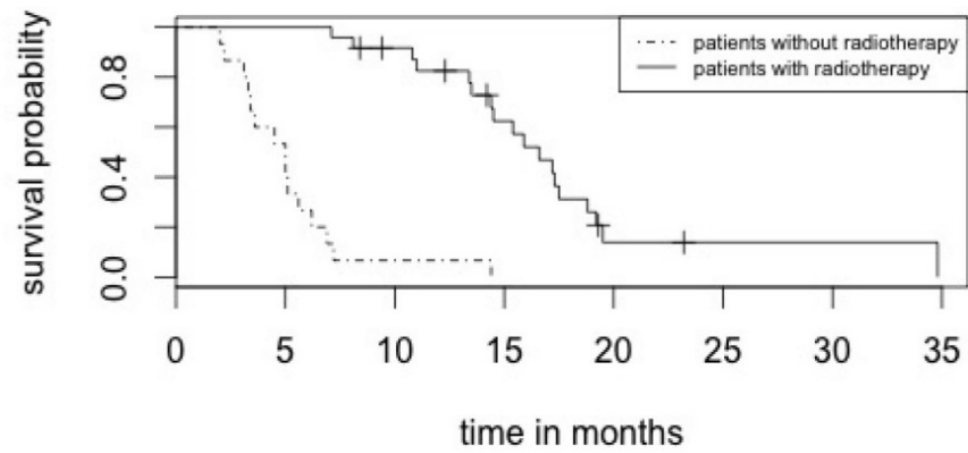

Number at risk

$\begin{array}{ccccccccc}\begin{array}{c}\text { Radiotherapy } \\ \text { after TEM/BEV }\end{array} & 24 & 24 & 20 & 12 & 2 & 1 & 1 & 0 \\ \begin{array}{c}\text { No radiotherapy } \\ \text { after TEM/BEV }\end{array} & 15 & 8 & 1 & 0 & 0 & 0 & 0 & 0\end{array}$

Figure 3. Overall survival for patients who received or did not receive radiotherapy after induction treatment by TMZ/BEV 
Upfront BEV and TMZ treatment is feasible with a good tolerance in most patients and provides a rapid and sometimes prolonged clinical benefit in some patients. Performance status was often rapidly improved and radiotherapy was then conducted under better conditions. All patients who started radiotherapy could complete it. TMZ/BEV induction permitted the patients with the worse prognosis to avoid a complex and long radiotherapy regimen. Given the heterogeneity of locally advanced GB, and technical difficulties to quickly start concomitant chemoradiotherapy, TMZ/BEV induction may represent a reasonable option in clinical practice. The role of BEV in the treatment of GB is currently highly discussed. Two large phase III studies (AVAglio and RTOG 0825) have not succeeded in demonstrating its role in overall survival, despite a significant increase in PFS [6, 7]. In a randomized phase II trial, neo-adjuvant and adjuvant BEV and irinotecan, combined with TMZ-radiation, had no impact on OS in the first-line treatment of unresectable GB [8]. However, patients with bulky GM, and/or severe neurological impairment (WHO performance status 3 or 4), and worst prognosis were not included in these trials. Few series have been devoted to locally advanced GB. A phase II trial of upfront therapy with $\mathrm{BEV}$, irinotecan (CPT-11), and TMZ prior to chemoradiation was done in 41 patients with unresectable, subtotally resected, and/or multifocal GB [13]. The authors reported a $22 \%$ response rate and $61 \%$ of patients had stable disease. Median PFS was 5.2-months and OS was 12 months. Not all patients received radiotherapy. Treatment was well tolerated. Our results are quite similar with $15 \%$ partial responses and $44 \%$ stable diseases. Median PFS and OS in patients treated by upfront TMZ-BEV were 8.4 months and 11 months respectively in an unselected cohort of 39 patients with locally advanced GB. The RPA V class regroups different clinical situations. Some patients have multifocal or bulky disease that could never be irradiated at a curative dose (60 Gy) in the tumor field. In the others, tumor volume is compatible with radiotherapy at a curative dose, but the tumor is located in a functional area and there is a severe neurological impairment at diagnosis. In such situations, bad general and neurological conditions hamper the planned radiotherapy. However, radiotherapy remains a major weapon against GB and must be used as often as possible to obtain increased survival [14]. In this sense, there is interest in reducing the tumor volume to facilitate radiotherapy. This goal was achieved in our series. Analysis of the tumor volume evolution in the selected 24 patients that received delayed radiotherapy demonstrates that upfront TMZ/BEV induced a global decrease of GTV,
CTV and PTV. Tumor volume decrease was obviously more dramatic in the patients that were in strict partial response according to RANO criteria. However, a less than $50 \%$ reduction in tumor and target volumes (GTV and CTV) still made radiotherapy feasible, even though it is considered as a stable response according to RANO criteria. Irradiated normal brain volume represented by a PTV/ whole brain ratio decreased from 0.33 to 0.25 in the whole cohort of irradiated patients.

The main limitation of the study is its retrospective nature. In this context, we were not able to provide molecular information of importance for prognosis and response to TMZ. MGMT methylation status was determined only in 17 patients. We observed a trend toward a better response to upfront TMZ-BEV in terms of CTV reduction in methylated $(\triangle \mathrm{CTV}=-84 \%)$ compared to non-methylated GB $(\Delta \mathrm{CTV}=-26.5 \%)$.

Median OS of patients treated with upfront TMZ-BEV were 11.0 months in the whole cohort of 39 patients and 15.0 months for the 24 patients that were finally treated by chemoradiation. To compare, the median OS was 14.6 months in the TMZ-radiotherapy arm from the EORTC pivotal study that established TMZ-radiotherapy as the standard treatment for GB (Stupp), 14.6 months in the AVAGLIO (Chinot) and 15.7 months in the RTOG studies, which unsucessfully explored the role of BEV on OS in first line treatment of GB. In these three studies, only patients with IK $>70$ were included and most were in RPA III and IV classes. In comparison, IK was $<70$ in $38 \%$ of our patients and $74 \%$ were in RPA class V. In total, TMZ/BEV induction seems to allow the majority of GB patients that are deleteriously impacted by poor performance status and neurological deficit, to complete a curative-intent radiotherapy, with a beneficial effect on their OS. In conclusion, TMZ/BEV induction often demonstrated a clinical activity in locally advanced GB, providing partial responses and significant levels of disease stabilization. TMZ-BEV induction reduced target volume delineation and rendered delayed radiochemotherapy feasible for a majority of patients. Further investigations are warranted through a randomized phase III trial devoted only to locally advanced GB.

\section{Importance of the study}

Standard of care is not defined in patients newly diagnosed with bulky glioblastoma and/or with severe neurological impairment, and the prognosis is poor. The present study reports the feasibility and efficacy of an induction treatment with temozolomide and bevacizumab (TEMO/BEVA) before delayed 
radiochemotherapy in order to alleviate the initial neurological impairment and/or to reduce the tumor volume.

\section{Acknowledgments}

We thank Zuzana Saidak for the paper revision. We thank Comité de la Somme de la Ligue contre le Cancer for its financial support.

\section{Competing Interests}

The authors have declared that no competing interest exists.

\section{References}

1. Li J, Wang M, Won M, Shaw EG, et al. Validation and simplification of the Radiation Therapy Oncology Group recursive partitioning analysis classification for glioblastoma. Int J Radiat Oncol Biol Phys. 2011;81:623-630.

2. Stupp R, Mason WP, van den Bent MJ et al. Radiotherapy plus Concomitant and Adjuvant Temozolomide for Glioblastoma. N. Engl. J. Med. 2005; 352:987-996.

3. Weller M, van den Bent M, Hopkins K, et al. EANO guideline for the diagnosis and treatment of anaplastic gliomas and glioblastoma. Lancet Oncol. 2014;15:395-403.

4. Jain RK, di Tomaso E, Duda DG et al. Angiogenesis in brain tumours. Nat. Rev. Neurosci. 2007; 8:610-622

5. Vredenburgh JJ, Desjardins A, Herndon JE 2nd, et al. Phase II trial of bevacizumab and irinotecan in recurrent malignant glioma. Clin Cancer Res. 2007;13:1253-9

6. Chinot OL, Wick W, Mason W et al. Bevacizumab plus Radiotherapy-Temozolomide for Newly Diagnosed Glioblastoma. N. Engl. J. Med. 2014; 370:709-722.

7. Gilbert MR, Dignam JJ, Armstrong TS et al. A Randomized Trial of Bevacizumab for Newly Diagnosed Glioblastoma. N. Engl. J. Med. 2014; 370:699-708.

8. Chauffert B, Feuvret L, Bonnetain F, et al. Randomized phase II trial of irinotecan and bevacizumab as neo-adjuvant and adjuvant to temozolomide-based chemoradiation with temozolomide-chemoradiation for unresectable glioblastoma: final results of the TEMAVIR study from ANOCEF†. Ann Oncol. 2014;25:1442-7.

9. Bleehen NM, Stenning SP. A Medical Research Council trial of two radiotherapy doses in the treatment of grades 3 and 4 astrocytoma. The Medical Research Council Brain Tumour Working Party. Br J Cancer. 1991;64:769-74.

10. Niyazi M, Brada $M$, Chalmers AJ et al. ESTRO-ACROP guideline "target delineation of glioblastomas." Radiother. Oncol. 2016; 118:35-42.

11. Assouline A, Huet de Froberville H, Calitchi E et al. Arcthérapie volumétrique modulée du glioblastome près d'organes à risque. Comparaison dosimétrique avec la radiothérapie conformationnelle tridimensionnelle. Cancer/Radiothérapie 2014; 18(5-6):596.

12. Wen PY, Macdonald DR, Reardon DA et al. Updated Response Assessment Criteria for High-Grade Gliomas: Response Assessment in Neuro-Oncology Working Group. J. Clin. Oncol. 2010; 28:1963-1972.

13. Peters KB, Lou E, Desjardins A, Reardon DA, Lipp ES, Miller E, Herndon JE 2nd, McSherry F, Friedman HS, Vredenburgh JJ. Phase II Trial of Upfront Bevacizumab, Irinotecan, and Temozolomide for Unresectable Glioblastoma. Oncologist. $2015 ; 20: 727-8$.

14. Walker MD, Green SB, Byar DP, Alexander E, Jr, et al. Randomized comparisons of radiotherapy and nitrosoureas for the treatment of malignant glioma after surgery. N Engl J Med. 1980;303:1323-1329. 